\title{
Educación salesiana para la ciudadanía desde la universidad para una sociedad líquida*
}

\author{
Salesian education for citizenship from the university to a liquid society \\ Roberto Alexander Damas Solórzano** \\ roberto.damas@udb.edu.sv
}

ISSN 1996-1642, Editorial Universidad Don Bosco, año 11, No.19, enero-junio de 2017, pp. 69-81.

Recibido: 25 de abril 2016. Aprobado: 29 de junio de 2016.

\begin{abstract}
Resumen
El artículo hace una reflexión desde un planteamiento pedagógico sobre cómo educar en ciudadanía en la universidad salesiana. Dicha reflexión implica una concepción de la educación como mecanismo para desarrollar la capacidad humana en una sociedad líquida en la cual la dimensión de ciudadana ha sido relevada por la de consumidores. Ante esto, la educación superior, y especialmente la presencia salesiana en la universidad, plantean una emergencia educativa, educar desde un modelo salesiano al ciudadano.
\end{abstract}

Palabras clave: capital humano, capacidad humana, sociedad líquida, universidad salesiana, educación, ciudadanía.

\section{Abstract}

This paper poses a reflection based on a pedagogical approach about how to educate in citizenship in a salesian university context. Such reflection implies an education understood as a mechanism for the development of human capacities in a liquid society in which the citizen dimension has been replaced for one of a consumer. To that point, Higher education, especially a salesian university, demands an educational emergency: to educate in citizenship under a salesian approach.

Keywords: human capital, human capacity, liquid society, salesian university, education, citizenship.

\footnotetext{
* Ponencia dictada con el nombre de Educar en la universidad salesianamente para una ciudadanía responsable en una sociedad líquida, el 20 de marzo de 2015 en la Universidad Pontificia Salesiana de Roma (UPS), en el Congreso Internacional de Pedagogía Salesiana denominado: Con Don Bosco educadores, de los jóvenes de nuestro tiempo, desarrollado en el Salesianum de Roma y dicha universidad.

** El autor es salesiano cooperador, máster en Políticas para la prevención de violencia juvenil en cultura de paz, Director de Pastoral Universitaria y docente de la Universidad Don Bosco.
} 


\section{La educación y el desarrollo de las capacidades humanas}

El arte de la educación es una vocación del educador y la educadora, es un compromiso coherente y competente para con los destinatarios; Don Bosco lo resumirá en una frase simple, pero no por ello poco profunda: "la educación es cosa del Corazón". Solo quien realmente ama, está dispuesto a llevar un proceso educativo capaz de humanizar, y de desarrollar las capacidades humanas en las personas.

La educación contribuye a desarrollar múltiples capacidades humanas: esquemas básicos de percepción, comprensión y acción, es decir la cosmovisión de la vida. De este modo se constituye en un eje fundamental del desarrollo humano, promoviendo la creciente autonomía de las personas, lo que desde la razón podríamos definir como la auto prevención y el desarrollo del criterio propio, mediante una percepción honesta de la realidad.

Un desarrollo a escala humana permite a las personas participar en mejores condiciones en la profundización y el mejoramiento de la calidad de vida, a través de una mejor relación con el ambiente, el trabajo, la profundización de la democracia y el uso racional de la ciencia y la tecnología, una alfabetización deontológica, desde la ética y la moral.

\section{El capital humano y la capacidad humana}

El primer concepto se concentra en el carácter de agentes [agency] de los seres humanos, que por medio de sus habilidades, conocimientos y esfuerzos, aumentan las posibilidades de producción, cuyo paradigma con frecuencia predomina en la educación superior, y que corre el riesgo de volverse una intención educativa tecnócrata; el segundo se centra en la capacidad de las personas para llevar el tipo de vida que consideran valiosa, e incrementar sus posibilidades reales de elección, ser un ciudadano por ejemplo, y compartir con los demás miembros de la sociedad valores y compromiso por el bien común y no solo del bien privado o individual (Sen, 1997).

Si bien es cierto que la educación desarrolla a la persona para una mejor producción de bienes, es claro que contribuye al mejoramiento del capital humano. Este mejoramiento puede optimizar la producción de la economía y aumentar el ingreso de la persona que ha sido educada. Pero aún con el mismo nivel de ingreso la persona puede beneficiarse de la educación por la posibilidad de leer, argumentar, comunicar, elegir con mayor información, ser tomada en cuenta por otros, de manera que los beneficios de la educación son mayores que su función de capital humano en la producción de bienes. La perspectiva más amplia de la capacidad humana puede abarcar -y valorar- estas funciones adicionales. Las dos perspectivas están íntimamente relacionadas aunque sean distintas.

Educación salesiana para la ciudadanía desde la universidad para una sociedad

70. liquida
El proceso de desarrollo de la persona por medio del hecho educativo, se da no solo con el desarrollo del capital humano, sino de la capacidad humana cuando se da énfasis a la expansión de la libertad humana para vivir el tipo de vida que la persona considera verdadera, una vida más libre, más digna. 
Es cierto que la prosperidad económica favorece que la gente lleve una vida más libre y realizada, también lo hacen una mayor educación, unos mejores servicios de salud y de atención médica y otros factores que influyen en las libertades efectivas de las que puede gozar una persona. Estos "desarrollos sociales" deben ser considerados directamente como "avances del desarrollo", puesto que contribuyen a tener una vida más larga, más libre y provechosa.

La capacidad humana nos recuerda el planteamiento de Adam Smith, en su polémica con David Hume, que cuando se considera a los seres humanos únicamente desde el punto de vista de su utilidad, se menosprecia la dignidad humana:

parece imposible que la aprobación de la virtud sea un sentimiento de la misma especie que la aprobación de un edificio cómodo o bien construido, o que no tengamos otra razón para elogiar a un hombre distinta de la que usamos para alabar un armario. (Smith, 1975).

El desarrollo de la capacidad humana por medio de la educación, desde un enfoque de aprendizaje ético, puede generar un cambio social, e ir más allá de un simple cambio económico. La capacidad humana no solo es un instrumento de la producción económica, sino también, y sobre todo del desarrollo social. Por ejemplo hay suficiente información empírica para sostener que la educación de la mujer reduce las condiciones de desigualdad con los hombres, dentro de la familia y la sociedad, pero además contribuye a reducir los embarazos precoces en adolescentes.

Por ello, estamos obligados a asegurar la formación para la profesionalidad donde el joven está implicado en un proceso de educación compleja en la que, además de las competencias relativas al trabajo, aprende los derechos y los deberes de ciudadanía activa; experimenta conductas sociales modeladas en la colaboración, en la responsabilidad individual y en la solidaridad; aumenta los propios conocimientos culturales; estructura la propia identidad de modo adecuado para integrarse en el tejido social y civil (Attard, 2014).

\section{Educar en una sociedad líquida}

Después de la modernidad "sólida" con sus dogmas, sus profetas, su culto al progreso y a la ciencia, con las grandes ideologías (cientifismo, positivismo, nazismo, marxismo, seguridad nacional) se enfrenta hoy, en el rico Occidente pero también en otras partes del mundo, con la modernidad "líquida" en la que nada es estable, nada es cierto, todo es ambiguo, móvil, inaprensible, precario, incontrolable. (Vitali, 2014)

Somos, escribe Zygmund Bauman, nómadas globales que nos sentimos incómodos en una sociedad individualizada, sola y asustada en un mundo fluctuante, líquido, incierto, sin ninguna vinculación, ni reglas, ni certezas, ni puntos de referencia, encerrados en la soledad del ciudadano global. 
Por doquier se multiplican los síntomas de alejamiento de las personas de la política y de la pérdida de interés por el funcionamiento del proceso político, desinterés que se experimenta también entre los jóvenes, ya que con frecuencia los procesos educativos no desarrollan programas y acciones pedagógicas, para educar en ciudadanía a las personas. Pero la política democrática no puede sobrevivir mucho tiempo ante la pasividad de los ciudadanos que se alimenta de la ignorancia y de la indiferencia política (Bauman, 2000). "Aquí comienza la corrupción de las personas y de las estructuras".

En una vida líquida no hay lugar ni para héroes ni para mártires, ya que no existe ninguna trinchera que defender y menos aún valores por los que dar la vida. En su lugar, saltan al proscenio del teatro las "celebridades", las estrellas, los nuevos héroes de la canción, del deporte, del cine y de las finanzas y porque no los súper políticos que prometen resolver los problemas de la sociedad por medio de apariciones espectaculares y populistas en las industrias culturales, 0 involucrados en corrupción y crimen organizado, pero con un ausentismo cínico en sus territorios.

Lo sólido se disuelve, y se está derritiendo. La modernidad líquida la constituyen los vínculos entre las elecciones individuales y los proyectos, y las acciones colectivas donde lo particular priva sobre lo público. Basta un ejemplo: en América Latina la inseguridad ha desatado un encierro de las personas en sus viviendas, a manera de caja fuerte, con alambre de púas, puertas con tres o más pasadores de seguridad, que generan una conducta de aislamiento ante los demás vecinos, y un silencio cómplice que tolera la violencia doméstica, porque es algo privado donde nadie se mete, Se aplica el dicho: "mientras no pase en mi casa, no me importa"; esto nos evidencia una clara falta de conducta ciudadana y del cuido del bienestar social, comunal y local. La principal técnica de poder es ahora la huida, el escurrimiento, la elisión, la capacidad de evitar en todo lo posible el involucramiento en acciones de ciudadanía activa.

La institucionalidad de los países como estados, está siendo suplantado por el poder económico y político global en las relaciones financieras, o guerras cuyo fin ya no es el control de territorio, sino el desmoronamiento de fronteras para las relaciones comerciales unidireccionales y desiguales, donde los estados son cada vez menos estados y ejercen menos control en sus territorios, ante ciudadanos pasivos e inertes, de la realidad local, nacional y global, debido, entre otras cosas, a un abuso del paradigma educativo funcionalista que solo se encarga de formar el capital humano.

Estamos ante la época de personas e instituciones zombis, que qunque están muertas, todavía están vivas. Ya lo afirma Bauman: dar una nueva forma (líquida) a las realidades y situaciones, es más fácil que mantenerlas en su estado original (sólido) (Bauman, 2000), La desintegración de la trama social y el desmoronamiento de las agencias de acción colectiva suelen señalarse con gran ansiedad y justificarse como "efecto colateral" anticipado de la nueva fluidez de un poder cada vez más móvil, escurridizo, cambiante evasivo,

Educación salesiana para 
fugitivo. Pero la desintegración social es tanto una afección como un resultado de la nueva técnica del poder, que emplea como principales instrumentos el descompromiso y el arte de la huida.

\section{Consumidores del siglo XXI y ciudadanos del siglo XVIII}

¿Consumidores y ciudadanos? Es la pregunta de Néstor García Canclini que nos invita a reflexionar sobre la conciencia ciudadana no solo de los jóvenes, sino de toda la sociedad en general, en un tiempo en que la actividad política se traslada de los mítines a la televisión, de las polémicas doctrinarias a confrontación de imágenes, y de la persuasión ideológica a las encuestas de marketing, todo ello nos hace sentir convocados como consumidores aun cuando se nos interpele como ciudadanos. Desaparecida la antigua figura de los ciudadanos con fuerte compromiso políico y social, se pone al frente otra nueva: la del "consumidor". "El ascenso del consumidor es la caída del ciudadano", (García, 1991).

En América Latina hay una crisis de espacios públicos, parques, zonas verdes, zonas de esparcimiento social, lugares de encuentro, etc. lo que imposibilita el ejercicio de la ciudadanía. Las relaciones entre los habitantes y entre el poder y la ciudadanía se materializan, se expresan en la conformación de las calles, las plazas, los parques, los lugares de encuentro ciudadano, en los monumentos. La ciudad entendida como sistema, de redes o de conjunto de elementos -tanto si son calles y plazas como si son infraestructuras de comunicación (estaciones de trenes y autobuses), áreas comerciales, equipamientos culturales es decir espacios de uso colectivo debido a la apropiación progresiva de la gente- que permiten el paseo y el encuentro, que ordenan cada zona de la ciudad y le dan sentido, que son el ámbito físico de la expresión colectiva y de la diversidad social y cultural. Es decir que el espacio público es a un tiempo el espacio principal del urbanismo, de la cultura urbana y de la ciudadanía. Es un espacio físico, simbólico y político, es allí donde se concretan y practican los deberes y las obligaciones que definen al ciudadano. (Borja \& Mauxi, 2000).

Los centros comerciales se han convertido en los nuevos espacios públicos, en los cuales se ejerce un tipo de "ciudadanía", determinada por el consumo y el poder adquisitivo, la dimensión de consumo, sustituye a la dimensión ciudadana. Los ciudadanos quieren ser consumidores, sin percatarse que depende de la medida en que puedan consumir efectivamente, es decir, la medida que cuenten con los recursos económicos para ello. El ámbito de realización como consumidores es el mercado, el consumo sustituye a la práctica de la ciudadanía.

La incerteza que atenaza a la sociedad moderna se deriva de la transformación de sus protagonistas, de ciudadanos en consumidores. La exclusión social ya no se basa solamente en el alejamiento del sistema productivo o en el hecho de "no poder comprar lo esencial para vivir", sino en "no poder comprar para sentirse parte de la modernidad, esta modernidad líquida". 
Reducir a las persona a consumidoras, significa privarla de sus derechos fundamentales, así como de derechos civiles y políticos no menos fundamentales, la definición de una persona como consumidora la deja a expensas del mercado ante el cual solo cuenta en tanto tenga los recursos económicos para mantener ese estatus, su definición ciudadana la convierte no solo en agente de deberes y derechos, sino en parte de un todo social más amplio en el cual se juega su realización como persona dentro del tejido social (Gonzáles, 2011).

Para las democracias en transición, como las de Latinoamérica, es fundamental retomar la educación como un proceso de formación ciudadana, ya que solo con un ciudadano responsable, la democracia puede desarrollarse en una sociedad determinada.

Pero, ¿qué es ciudadanía? La ciudadanía implica verse a uno mismo como persona autónoma y competente, con un conjunto de metas personales por realizar; y, al mismo tiempo, como miembro de una comunidad política legitimado para discutir y para involucrarse en los asuntos que competen a dicha comunidad.

Como capacidad de involucrarse y contribuir a la vida pública. En este sentido más amplio, la ciudadanía se refiere al compromiso personal adquirido ante situaciones de la vida pública. A menudo denominada ciudadanía activa, abarca un rango de actividades que van más allá de la participación electoral. Implica reconocer a las personas como sujetos capaces de proponer, exponer puntos de vista y tener injerencia en la realización de cambios en las diferentes esferas sociales y políticas (FOD, 2010).

\section{Educar salesianamente al ciudadano en la universidad}

No es común que la universidad sea un lugar en el que se aprenda un conjunto de saberes éticos y ciudadanos. Sin embargo, la educación universitaria de calidad no puede separar la formación profesional de la formación ciudadana, es la universidad como cuna de humanización, donde se vive y construye la ciudad, desde la comunidad académica universitaria.

En el actual contexto sociocultural, en medio de una sociedad líquida, de consumidores, la universidad es el óptimo espacio de aprendizaje en ciudadanía, no solo de carácter profesional y cultural en su sentido más amplio, sino también de carácter humano, y por ende ético y moral.

El sentido y la misión pública de la universidad no están determinados por el carácter público o privado de su titulación, sino en función de un conjunto de características que permiten concebirla -o no- como un espacio de aprendizaje

Educación salesiana para la ciudadanía desde la universidad para una sociedad

74. liquida ético que procura que sus titulados ejerzan futuras profesiones con la voluntad de contribuir a la formación de una sociedad inclusiva, digna y democrática: buenos cristianos y honestos ciudadanos. 
La educación superior salesiana promueve el desarrollo integral del joven mediante la asimilación y la elaboración crítica de la cultura, mediante la educación en la fe, con miras a la transformación cristiana de la sociedad', por medio de una propuesta de vida, que desde la fe, entra en dialogo con el conocimiento y la ciencia (IUS, 2012).

Hoy la formación de un buen profesional debe incluir su formación como ciudadano y como persona. Por ello la universidad es un espacio de construcción, no solo de conocimientos sino, de valores en el que los estudiantes que allí se formen puedan aprovechar al máximo los recursos que ella le ofrece.

Hoy la universidad salesiana es la que debe esforzarse por hacer más digna la sociedad, convirtiendo a sus estudiantes tanto en excelentes profesionales, como en ciudadanos y ciudadanas cada vez más cultos y críticos, por medio de la búsqueda de la verdad con rigor, la argumentación, el diálogo y la deliberación abierta, evitando fundamentalismos y dogmatismos (Martínez, 2006), por medio de una interacción entre educadores y educandos, en un ambiente de confianza y apertura, en un ambiente de familia.

Por ello la opción de la presencia salesiana en la Educación Superior se ha hecho con la voluntad de tener una incidencia educativa y cultural, que debe asegurar un impacto en la vida de los estudiantes que la frecuentan y una transformación en las sociedades en donde se encuentran. (IUS, 2012)

El elemento salesiano en educación ciudadana requiere de los educadores competencia para reconocer al otro -es decir al estudiante- en igualdad de derechos y condiciones, en tanto que personas, y para diseñar situaciones que les permitan discernir la intersubjetividad y la cotidianidad de la vida como elementos claves de su formación como personas. De este modo se podrá actuar luego, en consecuencia, más centrado en el educando que aprende que en el educador que enseña; más en los resultados del aprendizaje que en las formas de enseñar; más en el dominio de unas competencias procedimentales y actitudinales que en las informativas y conceptuales, lo que Don Bosco realizó de una manera más popular, por medio del ejemplo por parte del educador, a través de experiencias en el oratorio para reforzar la dimensión ética y moral de los jóvenes.

Es por ello que el educador tiene un compromiso moral con la institución, con la comunidad académica, a la que pertenece y la identidad salesiana de la misma, es un dinamizador de auténticas prácticas de enseñanza, de aprendizaje y de evaluación.

No falta entre el cuerpo del profesorado universitario el escepticismo sobre la educación en ciudadanía, valores y ética, manifestando que no es deber de la universidad dicha tarea, sino de la familia, la escuela y la comunidad, pero

1. Reglamento de la Sociedad de San Francisco de Sales 13. 
no de las instituciones de educación superior. El estudiante es una persona "adulta" que no precisa tanta tutela, y mucho menos de carácter ético. Para otros la universidad debe ocuparse de hacer bien sus tareas y no cuestiones como estas, ya que su misión es la de instruir y preparar para el ejercicio de una profesión, para la investigación o la docencia, y no la de educar o asumir tareas propiamente pedagógicas. También hay quienes piensan que todo esto significa introducir "moralismos" en la universidad, y afirman que eso es lo que no hace falta, olvidando que en la formación universitaria ahora las exigencias de los titulados reclaman cada vez más entrenamiento en la toma de decisiones de carácter ético, ya que los problemas actuales no pueden resolverse solo con respuestas científicas y técnicas, se necesitan respuestas sociales y éticas.

En este sentido la función clave del educador salesiano en la universidad es no dejar librado a la buena voluntad el hecho de que, para los estudiantes, la universidad sea un buen lugar de formación ciudadana. Ello implica cuestiones que van más allá de lo estrictamente docente, y así educar para reconocer y respetar la dignidad humana, entenderla como valor guía, que significa proponer un modelo de vida tanto individual como colectivo, que no solo facilite niveles de felicidad personal sino el ejercicio de una ciudadanía comprometida con el bien común y con el logro de una sociedad más equitativa (Martínez, Buxarrais, \& Bara, 2002). Es la tarea del educador salesiano hoy en la educación, y no solo en la universidad.

El educador salesiano en la universidad debe tratar que la relación de poder asimétrica conel estudiante le impida la enseñanza ética y moral: es en el ambiente de confianza, cercanía y respeto, ambiente de familia, donde el educador posee su mayor incidencia en los jóvenes. El educador es un profesional encargado de enseñar a aprender la ciencia, de enseñar a gestionar el conocimiento de una forma significativa y con sentido personal para el estudiante, de crear auténticos escenarios de enseñanza y aprendizaje. Es, además, el encargado de imprimir a los contenidos que enseña, el carácter ético que hará que el estudiante sea un experto profesional y un buen ciudadano. Se trata de que el docente se comprometa moralmente con su tarea formadora, es decir no se trata tanto de ser un experto competente, sino de querer serlo y comprometerse a serlo de forma que la acción docente no se limite al hecho de reproducir ciencia y de transmitirla, sino que sea una acción responsable con un compromiso ético hacia dicho conocimiento; por ello la formación salesiana de los miembros de la comunidad académica debe ser un esfuerzo pastoral constante y de calidad. El modelo de Aprendizaje-Servicio ${ }^{2}$, enfocado a las zonas de incidencia de la Universidad, puede servir como mecanismo de educación ciudadana desde las competencias de cada disciplina, al enfocar los trabajos, proyectos e investigaciones de cátedra, así como las institucionales, en beneficio de las personas y comunidades aledañas al centro de educación superior, en

Educación salesiana para la ciudadanía desde la universidad para una sociedad

76. liquida colaboración para resolver problemas y necesidades existentes.

Este modelo hace del territorio elegido un campo de práctica profesional, y de su realidad sociocultural una fuente de aprendizaje que favorece el desarrollo de 
la metacognición del estudiante, sumado a los aprendizajes dentro del aula, del laboratorio y el taller, que contribuyan a la formación del graduado, y la manera en cómo éste aborde su intervención profesional e incluya un compromiso social. De la misma manera pueden favorecer contextos de aprendizaje, situaciones y momentos de análisis y compresión hermenéutica de la realidad que se vive o se estudia, necesarios para que los estudiantes sean capaces de construir-adecuada, personal y autónomamente- sistemas de valores orientados a consolidar una sociedad basada en la dignidad de la persona, los estilos de vida y los valores propios de la democracia. Se trata de aprovechar los contenidos de aprendizaje propios de cada carrera y las situaciones de vida que la universidad ofrece de forma habitual y natural, para promover el desarrollo de aquellas dimensiones en la persona del estudiante como sujeto del aprendizaje ético, que le permita construirse racional y autónomamente en situaciones de interacción social.

Es importante comprender que el tratamiento pedagógico de lo ético, como elemento de ciudadanía, en el ámbito universitario no es solo cuestión de una modificación en el plan de estudios o de la incorporación de una nueva materia. Es, sobre todo, un cambio de perspectiva en relación con lo que hoy representa un buen nivel de formación universitaria, y con lo que debería significar el compromiso con lo público de una universidad que pretende formar buenos profesionales, buenos ciudadanos y ciudadanas, previniendo la actitud pasiva de los jóvenes estudiantes, en extremo interesados por la obtención del título para la competencia con otros en cuestiones estrictamente profesionales, a menudo alejadas, incluso contrarias, de las propuestas éticas relativas a los valores que les planteamos; además el contexto actual causa un nuevo malestar, no temporal, sino permanente. Al cambio incesante que caracteriza la sociedad y la cultura, se añade la debilidad de las instituciones que acompañan a los jóvenes. Aparece urgente e importante, la actitud responsable del educador salesiano y la solidez de su propuesta educativa (Attard, 2014), para un acompañamiento del joven en la universidad.

Debemos, por tanto avanzar en la dirección de una revalidación actualizada de la "opción socio-política-educativa" de Don Bosco. Esto significa también formar en una sensibilidad social y política, más allá del ejercicio del voto y la política partidaria, que lleva a invertir la propia vida como misión por el bien de la comunidad social, con una referencia constante a los inalienables valores humanos y cristianos, de manera que cada joven desarrolle las capacidades y las actitudes fundamentales para la vida en sociedad (Attard, 2014).

La misión de la universidad católica y salesiana consiste, por lo tanto, en ofrecer su aporte específico en este momento de la historia,

Ante todo con la competencia y el rigor científico unidos a una intensa vida espiritual, y sostenidos por ella, pero también con la creación de laboratorios culturales en los que se produzca un diálogo entre fe y cultura,

2. En Educación Superior las exigencias de aprendizaje-servicio se identifican como Compact-Campus y service Learning. Puede consultarse en www.compact.org y www.servicelearning.org. 
entre ciencia, filosofía y teología, y en los que la ética sea considerada una exigencia intrínseca de la investigación para un auténtico servicio al hombre. (Chávez, 2003).

La educación en los derechos humanos es indispensable como eje transversal de este proceso, en donde los valores como justicia, igualdad, libertad, solidaridad y dialogo sean apreciados como tales y denunciada su ausencia; un modelo formativo centrado en la responsabilidad y el conocimiento de los deberes y derechos, tanto en la profesión como en la ciudadanía. Implica otorgar un lugar central al diálogo y a la relación personalizada; cultivar la búsqueda constante de la verdad y la apertura a los valores; promover el crecimiento y la formación integral; propiciar la integración entre el conocimiento y la realidad concreta del trabajo; favorecer la participación responsable y solidaria en la vida comunitaria; promover el espíritu emprendedor y la capacidad de trabajo en equipo.

Universitariamente asumimos el estilo educativo salesiano, caracterizado por la confianza en la persona, la conformación de una comunidad que educa, la elaboración de una propuesta formativa integral, la creación de un ambiente hecho de relaciones cordiales y rico en experiencias de crecimiento, el protagonismo participativo de los jóvenes, la formación en el trabajo, la sensibilidad y la opción por los jóvenes más pobres, la presencia y acompañamiento del educador que se esfuerza por eliminar distancias y favorecer el encuentro (UDB, 2003).

\section{El asociacionismo un mecanismo de educación ciudadana}

El asociacionismo universitario y el voluntariado pueden servir como mecanismos de educación extra académicos, los jóvenes encuentran en ello un suelo firme para empezar con su construcción personal, que les sirve para su transformación, para asumir compromisos con ellos mismos, con la universidad y con su sociedad.

Se trata de un proyecto de vida, personal y grupal a la vez que expresa la vitalidad juvenil universitaria encausada por los grupos: culturales, deportivos, académicos y pastorales de la universidad salesiana. El criterio básico y fundamental es el mismo: la libertad. Nunca se obliga, ya que la participación es un acto voluntario del joven (Scaramussa, 1996), debido a que la coordinación y la conducción del grupo lo realizan los mismos jóvenes. Este empoderamiento lo recomendó el mismo Don Bosco: "Las compañías deben recomendarse y promoverse. Pero ustedes solo serán promotores, no directores; consideren tales cosas de los muchachos, bajo asesoría del educador" (Anadei, 1939).

De esta manera el Asociacionismo Salesiano Universitario, genera un espacio real para el joven y su participación en la comunidad educativa que le educa en esta dimensión ciudadana, para comprender de manera micro la dimensión

Educación salesiana para la ciudadanía desde la universidad para una sociedad

78. liquida macro del bien común y el bien individual, al hacerse cargo de su propia autoformación y autogestión del conocimiento, al servicio de los demás y de la universidad (Damas, 2014). 
El asociacionismo y la vida de grupo, en medio de una compleja sociedad líquida determinada por la fragmentación por medio de experiencias sociales y culturales, de masificación y de anonimato (Binder, 1991), ofrecen al joven un espacio de reconocimiento y de afirmación de la subjetividad, y del sentido unitario para la propia vida, el asociacionismo refuerza y respalda la participación de los jóvenes ante una sociedad adultocentrista ${ }^{3}$ (UNICEF, 2013) ya que sirve como espacio de comprensión de la sociedad, de preparación para insertar al joven de forma activa y transformadora en su contexto, de ejercicio para mantener la autonomía personal y el equilibrio en medio de los conflictos.

Este mecanismo permite que los jóvenes universitarios, organizados desde sus disciplinas y grupos de extensión, mantengan una relación horizontal, en un clima de familia con sus directores de escuela, docentes y las autoridades y funcionarios de la universidad, permitiéndoles desarrollar esas competencias para el ejercicio de una ciudadanía, por medio de la expresión de sus inconformidades, su propuestas, proyectos e iniciativas, como protagonistas de su propio proceso de aprendizaje, en un ejercicio democrático en donde desarrolla la convicción de no tener solo derechos, sino el cumplimiento exacto de sus obligaciones en beneficio de la sociedad y el tejido social al que pertenece.

\section{La gran tarea de vivir y actualizar el carisma salesiano en la universidad}

Como educadores e hijos e hijas de Don Bosco hoy, somos responsables de tratar de construir una sociedad diferente a través de la educación de la juventud y junto a ella, en esta sociedad líquida, en la cual nuestro carisma nos sirve como barca para poder educar a la juventud actual, con la tenacidad de Don Bosco para poder ver los signos de los tiempos, confiando que en los jóvenes siempre hay una semilla del reino de Dios (Fernández, 2014), y poder crear y fomentar sus valores y creencias, sus principios. Somos los llamados a defender el conocimiento de la opinión populista y barata, a defender la honestidad de la corrupción, a defender la paz de la violencia, a luchar por reducir la desigualdad, que ya Thomas Piketty cataloga de inmoral. Por ejemplo, en EEUU el $1 \%$ con mayor renta se queda con el $18 \%$ de la renta total: son los "super-ricos". (Piketty, 2014), y podríamos deducir el efecto cascada en América Latina de esa realidad, donde el lujo y la desigualdad se topan cruzando la calle, personas, y sobre todo jóvenes, que sobreviven con menos de un dólar al día, esperan de nosotros no la limosna sino que les reconozcamos su dignidad y trabajemos por defenderla, como lo dijo el Papa Francisco en su discurso ante la FAO en noviembre de 2014, a través de nuestra vocación educativa y con la competencia pedagógica.

3. Adultocentrismo: indica que existen relaciones de poder entre los diferentes grupos de edad que son asimétricas en favor de los adultos. Es decir, que estos se ubican en una posición de superioridad. Los adultos gozan de privilegios por el solo hecho de ser adultos porque la sociedad y su cultura así lo han definido. Por ejemplo, si un adolescente rompe un vidrio por error, recibe una sanción de parte del adulto (grito, reto, castigo o golpes), si este adulto comete el mismo error, no recibe castigo de parte del adolescente e incluso puede asumir que fue un accidente y, en el mejor de los casos, decir que tiene derecho a equivocarse. 
Se hace necesario asegurar una formación de calidad a los estudiantes, garantizando que los procesos de formación favorezcan el crecimiento personal y profesional, así como su inserción como ciudadanos en la sociedad. En concordancia con el estilo educativo salesiano, tal impacto deberá ser buscado a través de una propuesta académica de calidad, pero además por medio del acompañamiento y de la relación personal que debe caracterizar el proceso educativo (IUS, 2012).

La Universidad Salesiana está llamada en esta hora histórica a hacer que se comprenda y triunfe la prioridad del espíritu sobre la materia; la prioridad de las personas sobre las cosas; la prioridad de la ética sobre la técnica; la prioridad del trabajo sobre el capital; la prioridad del destino universal de los bienes sobre la propiedad privada; la prioridad del perdón sobre la justicia; la prioridad del bien común sobre los intereses personales (Chávez, 2003).

El rector mayor Don Ángel Fernández Artime nos invita en el Aguinaldo 2015 a pedir a Don Bosco que nos enseñe a leer los signos de los tiempos para ayudar a los jóvenes, para poder discernir la pregunta: ¿qué nos está diciendo y pidiendo Dios a través de ellos?, ya que de la respuesta pedagógica, depende nuestra salvación.

\section{Referencias}

Anadei, Á. (1939). Memorias Biográficas, Tomo X. Turín: CCS.

Attard, F. (2014). Cuadro de referencia de la pastoral juvenil salesiana. Roma: Editorial S.D.B.

Bauman, Z. (2000). Modernidad Líquida. Mexico: Fondo de Cultura Económica.

Binder, A. (1991). La Sociedad Fragmentada. Caracas.

Borja, J., \& Mauxi, Z. (2000). El Espacio público, ciudad y ciudadanía. Barcelona.

Canclini, N. (1995). Consumidores y Ciudadanos. México: Grijalbo.

Chávez, P. (2003). Europa: La universidad Católica y Salesiana debe llevar al continente la cultura de la cruz. La universidad Católica y Salesiana debe llevar al continente la cultura de la cruz. Roma: S.D.B.

Damas, R. A. (2014). El Asociacionismo Universitario Salesiano, como mecanismo de educación ciudadana. Diálogos(14), 55-63.

Fernández, Á. (Diciembre de 2014). Como Don Bosco, iCon los jóvenes, para los jóvenes! Aguinaldo Salesiano 2015. roma, Italia: S.D.B.

FOD, F. O. (2010). Manual de educación para la ciudadanía activa: Vol. I. Orientaciones teóricas y metodológicas. San José: cosude.

García, N. (1991). Consumidores y ciudadanos. Diálogos de la comunicación.

Gonzáles, L. A. (2011). ¿Consumidores o Ciudadanos? El Diario de Hoy.

IUS. (2003). Identidad de las Instituciones Salesianas de Educación Superior Protocollo. N. 03/0183. Roma: IUS.

Educación salesiana para la ciudadanía desde la universidad para una sociedad

80. liquida
IUS. (2012). Politicas. Roma: IUS.

IUS, V. A. (2012). PROGRAMA COMÚN 4. Roma: IUS.

Martínez, M. (2006). Formación para la ciudadanía y educación Superior. Revista Iberoamericana de Educación(42), 85-102. 
Martínez, M., Buxarrais, R., \& Bara, F. E. (2002). La universidad como espacio del aprendizaje ético. Revista lberoamericana de Educación N 29: Etica y Formación Universitaria, 5-24.

Piketty, T. (2014). Le capital au XXI siècle. Paris: Éditions du Seuil.

Scaramussa, T. (1996). El Sistema Preventivo de Don Bosco. San Salvador: UDB.

Sen, A. (1997). Capital Humano y Capacidad Humana. World development 25, 67-76.

Smith, A. (1975). The theory of moral sentiments. Clarendon: Oxford.

UDB. (2003). Ideario. San Salvador: UDB.

UNICEF. (2013). Superando el Adultocentrismo. Santiago de Chile: ONU.

Vitali, N. (2014). La dimensión salesiana en las IUS. La dimensión salesiana en las IUS: La Pastoral en la Universidad Salesiana (págs. 4-20). Sao Paulo: IUS. 